\title{
Commentary \\ Circulating cytokines and outcome prediction of burned children with concomitant inhalation injury
}

Pavlos M Myrianthefs and George J Baltopoulos

Athens University School of Nursing ICU at "KAT" Hospital, Nikis St, Kifissia, 14561, Greece

Corresponding author: Pavlos M Myrianthefs, pmiriant@nurs.uoa.gr

Published: 23 June 2008

Critical Care 2008, 12:155 (doi:10.1186/cc6920)

This article is online at http://ccforum.com/content/12/3/155

(c) 2008 BioMed Central Ltd

See related research by Gauglitz et al., http://ccforum.com/content/12/3/R81

\begin{abstract}
Being able to accurately predict probability of death is important for the intensivist. Serum cytokine levels parallel physiological derangements observed in critically ill patients and are used in commonly applied scoring systems and prediction models. Thus, serum cytokine based prediction models of outcome seem to be reasonable and of great interest. In this issue of Critical Care, Gauglitz and colleagues present their prediction equation for paediatric burn patients with concomitant inhalation injury. They found that IL-10 on admission, or IL-6 and IL-7 five to seven days later, may predict outcome in an excellent way. Increased mortality is observed as serum IL-6 and IL-10 levels increase and serum IL-7 levels decrease. However, the complexity of cytokine kinetics in critically ill patients and the variety of factors capable to affect circulating cytokines even in a subgroup of critically ill patients may affect the valitidy of the results. Also, serum cytokine based prediction models need to be compared to commonly applied prediction models based on clinical parameters. This will enable identification of the most suitable, accurate, cheapest, and easiest to use model to predict outcome.
\end{abstract}

In this issue of Critical Care, Dr Gauglitz and colleagues [1] present their prediction equation for outcome of burned children with concomitant inhalation injury based on serum cytokine measurements.

Prediction of outcome is very important in the intensive care unit (ICU) and, for this purpose, intensivists have created illness severity scores (Acute Physiology and Chronic Health Evaluation (APACHE), Simplified Acute Physiology Score (SAPS), and Mortality Probability Model (MPM)). These scores are calculated from data collected on the first ICU day and comprise two parts: the score itself, reflecting illness severity; and a probability model, which is an equation giving the probability of hospital death [2]. Accordingly, illness severity scores applicable for paediatric populations are widely used to assess severity and estimate probability of death $[3,4]$.

The probability of death after burns can be easily predicted on the basis of simple, objective clinical criteria: age greater than 60 years; more than $40 \%$ of body-surface area burned; and inhalation injury [5]. With regard to burn injury in children, only demographics and injury variables have been used to predict outcome $[6,7]$. In the later of these studies, an effort to take into account the effects of treatment on several variables to predict outcomes was attempted [7].

The activation of the host immune system and the release of inflammatory mediators have been linked to physiological derangements observed in burn injury and other inflammatory conditions, increasing according to illness severity and the progression of systemic inflammatory response syndrome to multiple organ failure. Thus, it has been assumed that increased physiological responses parallel the intensity of cytokine production and the development of multiple organ failure and death. Since the production or depression of several cytokines is related to physiological derangements commonly used in scoring systems, it seems reasonable to measure these circulating cytokines and use them as an additional tool to predict outcome.

In this regard, the article by Gauglitz and colleagues is original and of great interest [1].

The authors present their data on severely burned children with concomitant inhalation injury. They found that children who did not survive had the worst clinical characteristics, including lower $\mathrm{PaO}_{2} / \mathrm{FiO}_{2}$ ratios (arterial oxygen partial pressure/fraction of inspired oxygen), higher positive

ARDS $=$ acute respiratory distress syndrome; $\mathrm{FiO}_{2}=$ fraction of inspired oxygen; ICU $=$ intensive care unit; IL $=$ interleukin; $\mathrm{PaO}_{2}=$ arterial oxygen partial pressure; $\mathrm{PIP}=$ positive inspiratory pressure. 
inspiratory pressure (PIP), increased length of ventilator days and increased acute respiratory distress syndrome (ARDS) incidence. They also found that among 18 serum cytokines tested, IL-4, IL-6 and IL-13 were significantly higher on admission in non-survivors. Also, IL-10 was significantly higher on admission and on day 5 in non-survivors. On the other hand, non-survivors showed significantly lower IL-7 serum levels five to seven days post admission when compared to the survivor group.

Most importantly, using multiple logistic regression analysis, they created mortality prediction equations of burned children with concomitant inhalation injury using three serum cytokine values. They found that IL-10 level on admission, or IL- 6 and IL-7 levels five to seven days later, may predict outcome when used in these prediction equations. Increased mortality was observed as serum IL- 6 and IL-10 levels increased and serum IL-7 levels decreased.

This is an excellent work introducing serum cytokine measurements as indicators of physiological dearrangements in burned children with concomitant inhalation injury and using them to predict outcome. However, several issues need to be discussed.

At first, clinical data for non-survivors $\left(\mathrm{PaO}_{2} / \mathrm{FiO}_{2}, \mathrm{PIP}\right.$, length of ventilator days, ARDS incidence) were worse compared to survivors and this parallels cytokine measurements in nonsurvivors versus survivors for IL-4, IL-6, IL-7, IL10 and IL-13.

In our opinion, the investigators could also create a prediction model using easily obtained clinical parameters and without significant costs for comparisons, or compare their results with existing scoring systems for illness severity or death prediction models specifically developed for burn injury that use simple clinical parameters. Recently, another equation based on a very large database $(68,661$ patients) was published, taking into account seven variables, including age, total body surface area burned, inhalation injury, co-existing trauma, and pneumonia [8]. A comprehensive predictive model of burn mortality was created, providing superior predictive ability compared to previous models published $[6,7]$.

Another limitation of the study is that it included only a small number of patients and data from a single institution, which limit the validity of the results. Also, several other technical issues need to be clarified, including variability of the cytokine assays, sample processing, several physiological modifiers of cytokine production (tissue oxygenation, reactive oxygen species), and pharmacological modifiers of cytokine production. All these factors may affect cytokine induction and, consquently, serum cytokine levels.

Another study [9] in the past tried to correlate illness severity scores and plasma pro-inflammatory concentrations in critically ill ICU patients. The authors concluded that plasma cytokine concentrations fluctuate in serious illness and have a poor correlation with derangement of whole body physiology in seriously ill patients. The investigators concluded also that the daily measurement of plasma cytokine concentrations is not going to be clinically helpful in the ICU except possibly in specific subgroups of patients, such as those with sepsis. However, more recent data suggest that the use of a multiple cytokine assay platform allows identification of distinct cytokine profiles associated with sepsis severity, evolution of organ failure and death [10]. Also, in unselected critically ill patients, cytokine levels on ICU admission were independent outcome predictors for the presence and degree of organ dysfunction [11]. In the subgroup of septic patients, IL-6 was found to be the sole variable determining outcome. The authors also wondered whether cytokine measurements should be introduced in clinical practice for outcome prediction, and particularly in critically ill septic patients.

Medicine is an evolving science and art interested in death prediction in the ICU. Since the recognition by Holmes in 1860 [12] that the extent of injury is involved in determining burn outcome, age and other clinical variables have been added to better predict outcome [5-8]. Together with advances in computer science and statistical methods, serum cytokine measurements may be a new element in predicting outcome in critically ill patients in the 21 st century $[1,10,11]$. However, we should keep in mind the complexity of cytokine kinetics in critically ill patients and the variety of factors affecting circulating cytokines and that serum cytokine based prediction models need to be compared to commonly applied prediction models based on clinical parameters. This will allow us to better identify the most suitable, accurate, cheapest, and easiest to use model to predict outcome.

\section{Competing interests}

The authors declare that they have no competing interests.

\section{References}

1. Gauglitz G, Finnerty C, Herndon D, Mlcak R, Jeschke M: Are serum cytokines early predictors for the outcome of burn patients with inhalation injuries who do not survive? Crit Care 2008, 12:R81.

2. Le Gall J-R: The use of severity scores in the intensive care unit. Intensive Care Med 2005, 31:1618-1623.

3. Pollack MM, Ruttimann UE, Getson PR: Pediatric Risk of Mortality score. Crit Care Med 1988, 16:1110-1116.

4. Slater A, Shann F, Pearson G; Pediatric Index of Mortality (PIM) Study Group: PIM 2: a revised version of the Paediatric Index of Mortality. Intensive Care Med 2003, 29:278-285.

5. Ryan C, Schoenfeld D, Thorpe W, Sheridan R, Cassem E, Tompkins R: Objective estimates of the probability of death from burn injuries. N Engl J Med 1998, 338:362-366.

6. Wolf SE, Rose JK, Desai MH, Mileski JP, Barrow RE, Herndon DN: Mortality determinants in massive pediatric burns. An analysis of 103 children with $>$ or $=80 \%$ TBSA burns $(>$ or $=$ 70\% full thickness). Ann Surg 1997, 225:554-565.

7. Spies M, Herndon DN, Rosenblatt JI, Sanford AP, Wolf SE: Prediction of mortality from catastrophic burns in children. Lancet 2003, 361:989-994.

8. McGwin G Jr, George RL, Cross JM, Rue LW: Improving the ability to predict mortality among burn patients. Burns 2008, 34:320-327. 
9. Friedland JS, Porter JC, Daryanani S, Bland JM, Screaton NJ, Vesely MJ, Grifin GE, Bennet ED, Remick DG: Plasma proinflammatory cytokine concentrations, Acute Physiology and Chronic Health Evaluation (APACHE) III scores and survival in patients in an intensive care unit. Crit Care Med 1996, 24: 1775-1781.

10. Bozza FA, Salluh JI, André M Japiassu AM, Soares M, Assis EF, Gomes RN, Bozza MT, Castro-Faria-Neto HC, Bozza PT: Cytokine profiles as markers of disease severity in sepsis: a multiplex analysis. Crit Care 2007, 11:R49-56.

11. Dimopoulou I, Orfanos S, Kotanidou A, Livaditi O, GiamarellosBourboulis E, Athanasiou C, Korovesi I, Sotiropoulou C, Kopterides P, Ilias I, Kanellakopoulou K, Armaganidis A: Plasma pro- and anti-inflammatory cytokine levels and outcome prediction in unselected critically ill patients. Cytokine 2008, 41: 263-267.

12. Holmes T: A System of Surgery, Theoretical and Practical. London: JW Parker; 1860. 